\title{
Epigenome rejuvenation: HP1 $\beta$ mobility as a measure of pluripotent and senescent chromatin ground states
}

Received

21 November 2013

Accepted

17 March 2014

Published

25 April 2014

Correspondence and requests for materials should be addressed to M.M. (maria. manukyan@bioss.unifreiburg.de) or P.B.S. (prim.singh@charite. de)

\author{
Maria Manukyan' \& Prim B. Singh ${ }^{2}$
}

\begin{abstract}
${ }^{1}$ Albert-Ludwigs-Universität Freiburg, BIOSS Centre for Biological Signalling Studies, Schänzlestrasse 18, 79104 Freiburg, Germany, ${ }^{2}$ Fächerverbund Anatomie, Institut für Zell- und Neurobiologie, Charite - Universitätsmedizin, 10117 Berlin, Germany.
\end{abstract}

We measured the dynamics of an essential epigenetic modifier, HP1 $\beta$, in human cells at different stages of differentiation using Fluorescence Recovery After Photobleaching (FRAP). We found that HP1ß mobility is similar in human embryonic stem cells (hES) and iPS cells where it is more mobile compared to fibroblasts; HP1ß is less mobile in senescent fibroblasts than in young (dividing) fibroblasts. Introduction of "reprogramming factors", Oct4, Sox2, Klf4, cMyc and Lin28, into senescent fibroblasts and measuring the changes in HP1 $\beta$ mobility as reprogramming proceeds shows that the mobility of HP1 $\beta$ in senescent cells increases and by day 9 is the same as that found in young fibroblasts. Thus the dynamics of a key epigenetic modifier can be rejuvenated without de-differentiation through an embryonic stage. Future work will test whether other aspects of cellular physiology that age can be so rejuvenated without de-differentiation.

omatic cell nuclear transfer $(\mathrm{SCNT})^{1}$ has shown that a newborn clone can be derived from an old, differentiated, cell ${ }^{2,3}$. Likewise, senescent fibroblasts can be de-differentiated into a stable pluripotent ES-cell-like state (iPS cells $)^{4}$ using 'reprogramming factors' and then re-differentiated back to fibroblasts that have lost the senescent phenotype and have acquired the characteristics of young fibroblasts ${ }^{5}$. Based on these findings we have proposed that SCNT and iPS cell technology could be used to rejuvenate senescent cells without going through an embryonic stage because "developmental reprogramming" to the embryonic (ES and iPS cell) state may be molecularly separated from "age reprogramming". A key aspect of age reprogramming concerns the epigenome, which includes the DNA modifications, histone modifications and chromatin structures that regulate the expression, integrity and organisation of the genome ${ }^{6,7}$; during age reprogramming age-related changes in the epigenome of an old cell are reprogrammed to a youthful one found in a young cell by a process we have termed epigenetic rejuvenation ${ }^{8,9}$.

Chromatin is the natural environment of nearly all eukaryotic genes and is known to undergo changes during ageing ${ }^{10,11}$. Cytological examination has revealed that chromatin exists in two distinct states of compaction called euchromatin and heterochromatin ${ }^{12}$. Heterochromatin represents the dense compartment and its formation is thought to be regulated by a number of factors including transcription factor binding ${ }^{13}$, histone modification ${ }^{14,15}$, DNA methylation ${ }^{16}$ alteration of nucleosome positioning ${ }^{17}$, transcription of repetitive DNAs ${ }^{18,19}$ and the binding of non-histone chromosomal proteins such as Heterochromatin Protein $1(\mathrm{HP} 1)^{20}$. HP1 proteins are evolutionarily conserved proteins whose presence, along with methylated lysine 9 of histone $\mathrm{H} 3(\mathrm{Me}(3) \mathrm{H} 3 \mathrm{~K} 9)$, is a conserved characteristic of constitutive heterochromatin in organisms as diverse as fission yeast and $\operatorname{man}^{21}$. In mouse and man there are three HP1 isotypes, termed HP1 $\alpha, \mathrm{HP} 1 \beta$ and HP1 $\gamma^{22,23}$. The three mammalian isotypes exhibit a high degree of sequence and 3-D structural organisation similarity, but both antibody localisation studies and mutational analysis in mice have shown that they have non-redundant functions, with HP1 $\beta$ being essentia ${ }^{24}$. Notably, HP1 proteins are found at telomeric heterochromatin as part of the shelterin complex that maintains the structural integrity of the telomere "cap"25. Erosion of the telomeres is known to take place during ageing and the short telomeres of old cells can be lengthened by passage through an embryonic iPS cell stage ${ }^{26}$. HP1 proteins are components of senescence-associated heterochromatin foci (SAHF) that are thought to sequester proliferation-promoting genes as cells exit the cell cycle and enter a state of cellular senescence ${ }^{27,28}$. Cellular senescence can be induced by several methods, including oncogene induced senescence ${ }^{29}$, combined interferon gamma and TNF treatment of cells $s^{30}$ and replicative exhaustion ${ }^{31}$. Of these, induction of senescence by replicative exhaustion has been used as a well-known in vitro model for studying ageing at the cellular and molecular level ${ }^{31}$. HP1 proteins can also regulate gene activity locally, both positively and negativel $y^{32}$, and are known to bind to the 
Oct $3 / 4^{33}$ and Nanog $^{34}$ genes, which are key components of the pluripotency pathway in mammalian ES cells ${ }^{35,36}$. That HP1 proteins are part of the shelterin complex and SAHF and are likely involved in regulation of pluripotency genes has led to the suggestion that study of these proteins might provide insight into the molecular mechanisms underpinning epigenetic rejuvenation ${ }^{8,9}$.

HP1 proteins were one of the first proteins to be used in noninvasive FRAP studies to probe chromatin protein interactions in the living cell ${ }^{37}$. Numerous FRAP studies, in conjunction with kinetic modelling, have shown that at steady state equilibrium the nuclear HP1 pool can be separated into three kinetic fractions: a highly mobile "fast" fraction that freely diffuses through the nucleoplasm, a less mobile "slow" fraction that transiently binds to the HP1 ligand, $\mathrm{Me}(3) \mathrm{H} 3 \mathrm{~K} 9$, and a smaller immobilised HP1 fraction whose ligand(s) is not known ${ }^{38-44}$. Studies in the mouse have shown that the mobility of HP1 is dependent upon the differentiation state of the cell with HP1 being "hyper" dynamic in undifferentiated ES cells compared to the same cells after $24 \mathrm{hrs}$ of LIF withdrawal or differentiation into neuronal progenitors ${ }^{45}$. HP1 mobility in hES, human iPS cells and in senescent cells has not been determined.

Here we show, for the first time, that HP1 $\beta$ mobility is similar in hES and human iPS cells and is more mobile compared to primary human fibroblasts. Furthermore HP1 $\beta$ in senescent cells is significantly less mobile than in young (dividing) fibroblasts. To test the central idea of epigenetic rejuvenation we have measured, using FRAP, the dynamics of HP1 $\beta$ during the reprogramming of senescent fibroblasts. To do this we developed a construct based on the "piggyBac" construct ${ }^{46}$ that expresses five "reprogramming factors" along with a marker gene, dsRed, whose expression enables reprogramming to be followed at the single cell level. Introduction of the construct into senescent fibroblasts and following the changes in HP1 $\beta$ mobility as reprogramming proceeds shows that the mobility of HP1 $\beta$ in senescent cells increases and by day 9 is the same as that found in young fibroblasts. This occurs without any overt changes in the differentiated phenotype of the fibroblast. Thus the dynamics of a key epigenetic modifier can be rejuvenated without dedifferentiation indicating that epigenetic rejuvenation, with respect to HP1 $\beta$ mobility, has been achieved using iPS cell technology.

\section{Results}

HP1 $\beta$ has the same mobility in hES and iPS cells and is less mobile in LF1 primary human fibroblasts. Using the five-factor "piggyBac" vector $^{46}$ we reprogrammed LF1 primary human fibroblasts to produce 10 iPS cell lines of which one, line \#2.4, was characterised further. Line \#2.4 had a normal chromosomal complement (Fig. S1a), was alkaline phosphatase positive (Fig. S1b), showed reduced CpG methylation of the Oct4 and Nanog genes compared to the parental LF1 fibroblasts (Fig. S1c), expressed six different pluripotency markers in common with H9 cells (Fig. S1d) and could differentiate into all three germ layers (Fig. S1e). Endogenous HP1 $\beta$ and $\mathrm{Me}(3) \mathrm{K} 9 \mathrm{H} 3$ co-localised in hES and iPS line \#2.4 nuclei (Fig. 1a; top two rows) and the protein levels of HP $1 \alpha$, HP $1 \beta$ and HP1 $\gamma$ were similar in hES and the iPS line \#2.4 cell extracts (Fig. 1b). We introduced HP1 $\beta$-GFP into H9 and iPS line \#2.4 by transient lipofection and measured the FRAP of HP1 $\beta$-GFP foci. The recovery data were similar and showed no significant difference (Table 1; Fig. 2a; $\mathrm{p}=0.85$ ).

We next measured HP1 $\beta$ mobility in the parental LF1 primary human fibroblast cell line that was used for reprogramming into iPS cells. Measurement of the HP1 $\beta$ mobility in LF1 nuclei after transient lipofection showed that the recovery data was significantly different to that for hES and iPS line \#2.4 (Table 1; Figs. 2b and 2c; both $\mathrm{p}<0.0001)$.

HP1及 is less mobile in senescent compared to young LF1 fibroblasts. Young LF1 fibroblasts were positive for the proliferation marker Ki-67 and negative for SA- $\beta$-galactosidase activity (Fig. S3a). LF1 fibroblasts were passaged until they reached senescence and had exhausted their proliferative capacity. Senescent LF1 cells were positive for SA- $\beta$-galactosidase activity and negative for Ki-67 (Fig. S3b). In both young and senescent LF1 cells endogenous HP1 $\beta$ co-localised with $\mathrm{Me}(3) \mathrm{K} 9 \mathrm{H} 3$ (Fig. 1a, bottom two rows). When the protein levels of HP1 $\alpha, \mathrm{HP} 1 \beta$ and HP1 $\gamma$ in cellular extracts from senescent LF1 cells were compared to young (dividing) LF1 cells it showed that the levels were lower (Figures $1 \mathrm{~b}$ and S2b). The histone $\mathrm{H} 3$ control levels also decrease in senescent cells indicating that the nuclear:cytoplasmic ratio decreases in senescent cells, which may be related to the known increase in overall size of cells as they enter cellular senescence ${ }^{47}$. As shown in Table 1 measurement of the HP1 $\beta$ mobility in senescent LF1 nuclei after transient lipofection showed that the recovery data was significantly different to that for the young LF1 cells (Table 1; Fig. 2d; $\mathrm{p}<0.0001$ ).

Rejuvenation of HP1ß mobility in senescent LF1 cells by introduction of reprogramming factors. In order to be able to measure the mobility of HP1 $\beta$ during the reprogramming process we generated LF1 fibroblast cell lines that stably expressed HP1 $\beta$-GFP (LF1/ HP $\beta-$ GFP fibroblasts; Fig. S4). We chose a line that expressed "low" levels of HP1 $\beta-$ GFP for our experiments (see Fig. S4); it has previously been shown that HP1-GFP dynamics is insensitive to expression levels ${ }^{40,45}$. Use of LF1/HP1 $\beta-$ GFP fibroblasts avoided the need to transiently transfect HP1 $\beta-$ GFP into cells at various times during the reprogramming process. LF1/HP1 $\beta-$ GFP fibroblasts were passaged until they reached senescence (Fig. S3c). To follow the reprogramming of LF1/HP1 $\beta-$ GFP senescent fibroblasts after introduction of the 5-factor "piggyBac" vector we engineered the construct so that it expressed the $d s R e d$ marker gene along with the 5 reprogramming factors (see material and methods, Fig. S5). The newly-constructed vector, called "dsRed-OSKML", enabled the identification of single cells that had taken up the vector. HP1 $\beta$ mobility was measured in senescent LF1/ HP1 $\beta$-GFP fibroblasts on day 0 (just before dsRed-OSKML was introduced by lipofection) and every three days (days 3, 6, 9 and 12) after lipofection of dsRed-OSKML. As shown in Figs. 3a-c the recovery curves for days 0,3 and 6 were different to young (dividing) control LF1/HP1 $\beta$-GFP fibroblasts $(\mathrm{p}<0.0001)$. In particular, the mean $\mathrm{T}_{1 / 2}$ halftime values for the "slow" fraction were over 5 seconds for days 0 through to 6 compared to the young control LF1/ HP1 $\beta$-GFP fibroblasts which have a mean "slow" halftime of 4.4 seconds (Table 2). We also observed that the values for $\%$ immobile fraction varied considerably over the same time scale, with day 3 showing a mean value of $6 \%$ compared to $16 \%$ and $20 \%$ for the controls, young and senescent LF1/HP1 $\beta-$ GFP fibroblasts respectively. On day 9 the HP1 $\beta$ recovery data showed that HP1 $\beta$ was more mobile and was similar to that of control young LF1/ HP1 $\beta-$ GFP fibroblasts (Fig. $3 \mathrm{~d}, \mathrm{p}=0.12$ ). There was also a reduction in SA $\beta$-galactosidase staining of the cells at day 9 with $49 \%$ of cells being positive for $\beta$-galactosidase compared to $90 \%$ for day 0 (Fig. S3d). Measurement of HP1 $\beta$ mobility on day 12 showed that it was less mobile and now showed similar kinetics to that for days 0 through to 6 and the recovery curve was again different to the control LF1/HP1 $\beta$-GFP fibroblasts (Fig. 3e; $\mathrm{p}<0.0001$ ).

\section{Discussion}

The hallmarks of ageing ${ }^{11}$ are reversible. Old cells can be rejuvenated when they are reprogrammed by $\mathrm{SCNT}^{2,3}$ or via iPS cell induc$\operatorname{tion}^{5,26,48}$. Both methods require the passage of the old cell through an embryonic (ES or iPS cell) stage. By contrast we have proposed that "developmental reprogramming" to an embryonic stage can be separated from "age reprogramming" where the aged phenotype is reverted back to a youthful one; an old cell is rejuvenated while maintaining its specialised, differentiated, functions ${ }^{8,9}$. In this work 
a.
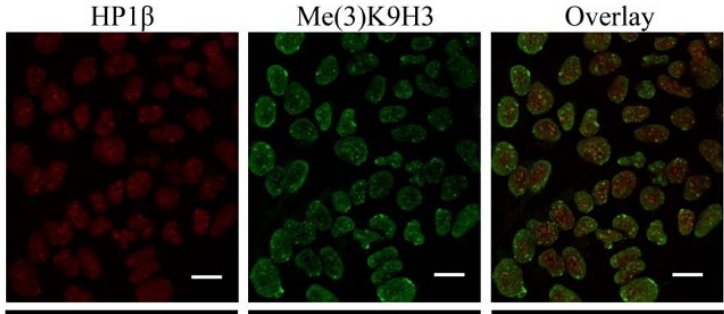

H9
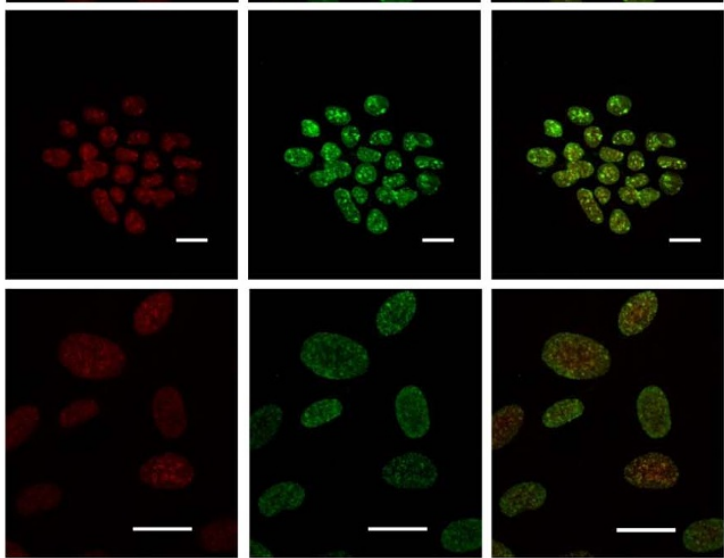

iPS
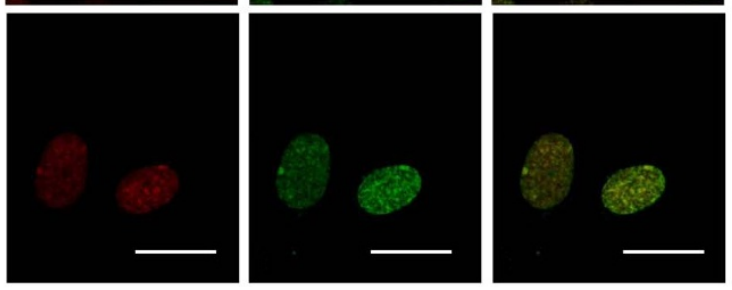

LF1-sen

b.

H9 iPS

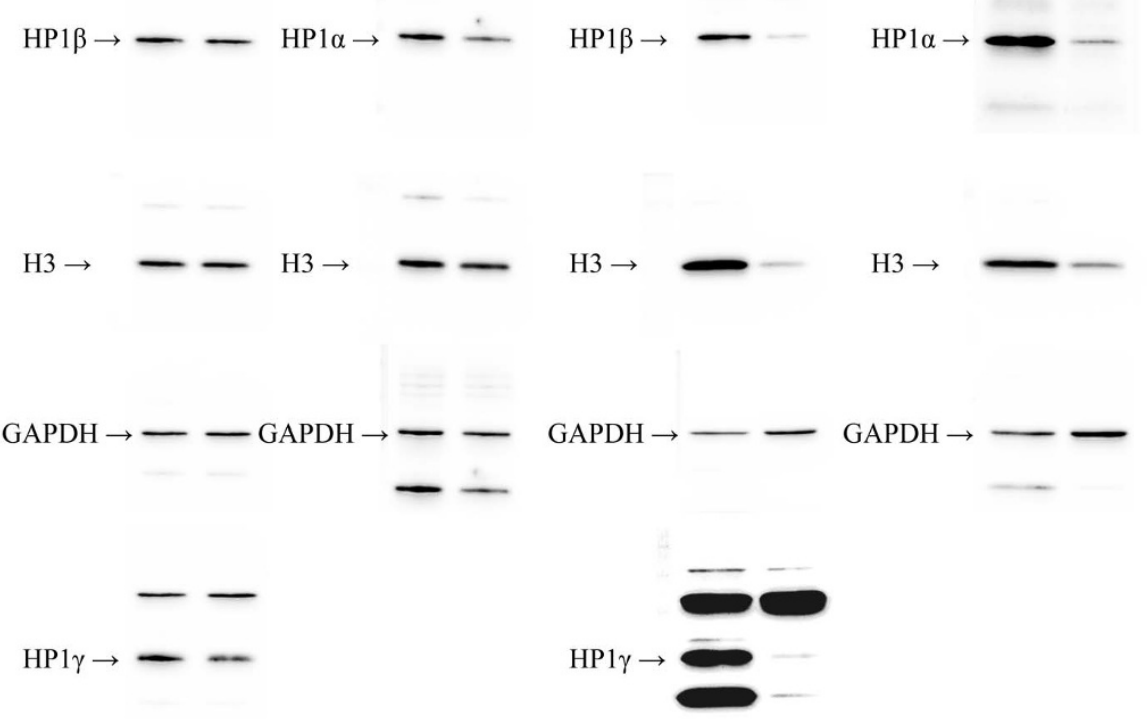

Figure 1 Nuclear localisation and detection of HP1 $\beta$ in H9 hES, iPS, LF1 and senescent LF1 cells. (a) The left-hand column depicts the distribution of $\mathrm{HP} 1 \beta$ (red) and the middle column shows the distribution of tri-methylated lysine 9 of histone $\mathrm{H} 3 \mathrm{in}(\mathrm{Me}(3) \mathrm{K} 9 \mathrm{H} 3)$ (green). The merged overlay of the images in the left-hand and middle columns is given in the right hand column. For all cells types (going top to bottom) H9, iPS, LF1 and LF1 senescent cells the enriched foci of $\mathrm{HP} 1 \beta$ and $\mathrm{Me}(3) \mathrm{K} 9 \mathrm{H} 3$ overlap and are seen as yellow foci in the right-hand column. The scale bar in each panel is $20 \mu \mathrm{m}$. (b) The levels of HP $1 \alpha, \mathrm{HP} 1 \beta$ and HP $1 \gamma$ were similar in H9 hES and the iPS line \#2.4 cell extracts (protein loading amount $20 \mu \mathrm{g}$ ). The levels of HP1 $\alpha$ and HP1 $\beta$ were similar in LF1 cell extracts with HP1 $\gamma$ showing slightly greater levels. The protein levels of HP1 isotypes appeared higher in young LF1 cells compared to senescent LF1 cells (protein loading amount $30 \mu \mathrm{g}$ ). However, once corrected for the endogenous H3 levels, which reflected the amount of nuclear proteins isolated in the cell extracts, the protein levels of the three HP1 isotypes in both young and senescent cell extracts were similar. Each blot was probed multiple times; for uncropped blots and order of probing please see Supplementary Fig. S2. 
Table 1 | FRAP analysis of HP1 $\beta$ mobility in H9 hES, iPS, young LF 1 and senescent $L F 1$ cells. The parameters for $\mathrm{H} 9 \mathrm{hES}$ and $\mathrm{iPS}$ cells are not significantly different to each other $(p=0.85)$. The recovery parameters for LF1 cells are significantly different ( $p<0.0001)$ from parameters for iPS and H9 hES cells. Senescent LFl cells have longer halftimes for the "fast" and "slow" fractions compared to LF1 cells $(p<0.0001)$

\begin{tabular}{lcccc} 
& H9 & iPS & LF1 & LF1 senescent \\
\hline $\mathrm{T}_{1 / 2 \text { fast }}(\mathrm{s})$ & $0.27 \pm 0.1$ & $0.3 \pm 0.09$ & $0.33 \pm 0.1$ & $0.45 \pm 0.1$ \\
$\mathrm{~T}_{1 / 2 \text { slow }}(\mathrm{s})$ & $3.38 \pm 0.6$ & $3.6 \pm 0.48$ & $3.8 \pm 0.3$ & $5.2 \pm 0.6$ \\
Free $(\%)$ & $32 \pm 5.1$ & $33 \pm 3.6$ & $26 \pm 2.7$ & $26 \pm 2.3$ \\
Bound $(\%)$ & $50 \pm 5.1$ & $49 \pm 3.6$ & $57 \pm 2.7$ & $57 \pm 2.3$ \\
$\mathrm{f}_{\text {im }}(\%)$ & $18 \pm 5.8$ & $18 \pm 3.7$ & $17 \pm 2.9$ & $17 \pm 3$ \\
\hline
\end{tabular}

we have tested whether these two apparently seamless aspects of reprogramming - developmental and age reprogramming - can be disentangled using as our experimental approach the mobility of the essential non-histone protein, HP1 $\beta^{20}$.

HP1 $\beta$ has the same kinetic parameters in hES and human iPS cells and is significantly more mobile in these embryonic cell types than in the LF1 fibroblasts from which the iPS cells were derived. This extends the work in the mouse, which has shown that HP1 is "hyper" dynamic in murine ES cells compared to differentiated progeny ${ }^{45}$. Taken together with our work on human cells these data indicate that the higher mobility of HP1 proteins in ES cells might serve as a parameter that characterises the epigenetic chromatin "ground state" of ES cells. While experimentally more involved than using antibodies for stem cell markers, measurement of HP $1 \beta$ mobility after transient transfection of HP1 $\beta$-GFP into presumptive ES/iPS cells might provide a measure of the degree of reprogramming achieved and, further, whether the high mobility of HP1 $\beta$ characteristic of ES cells has been attained.

HP1 $\beta$ mobility in dividing, Ki-67 positive, LF1 cells is significantly greater than in non-dividing, SA $\beta$-galactosidase positive, senescent LF1 cells that have exited the cell cycle. Comparison of $T_{1 / 2}$ for the "slow" fraction across the different cell types studied shows that senescent cells have a mean halftime that is over five seconds whereas all other halftimes are less than four seconds (Table 1). The decreased mobility of HP1 $\beta$ represents, we suggest, the senescent epigenetic chromatin "ground state" of old, non-dividing cells and provides a marker against which "epigenetic rejuvenation" upon reprogramming of senescent cells by iPS cell induction can be measured. Introduction of five reprogramming factors into senescent LF1 cells showed that the mobility of HP1 $\beta$ increased in senescent cells and reached the levels found in young LF1 cells by day 9 ; there was also a decrease in SA $\beta$-galactosidase positive cells on day 9 (Figure S3d). a.

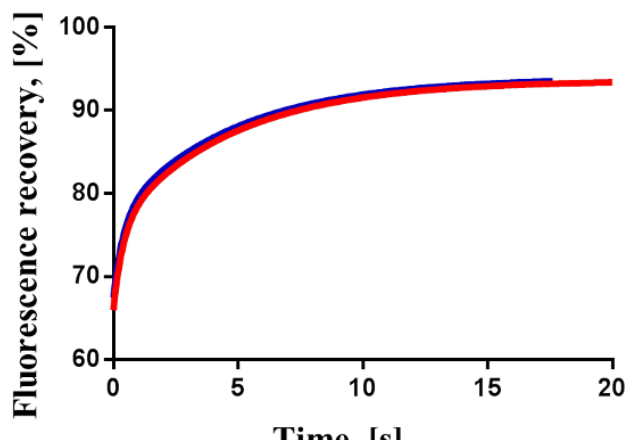

Time, $[\mathbf{s}]$

c.

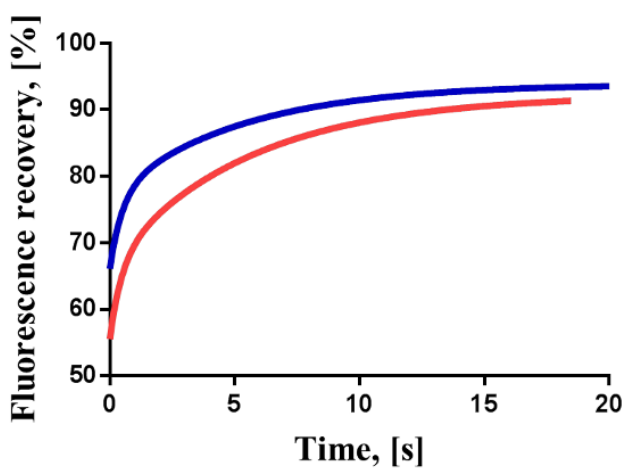

b.

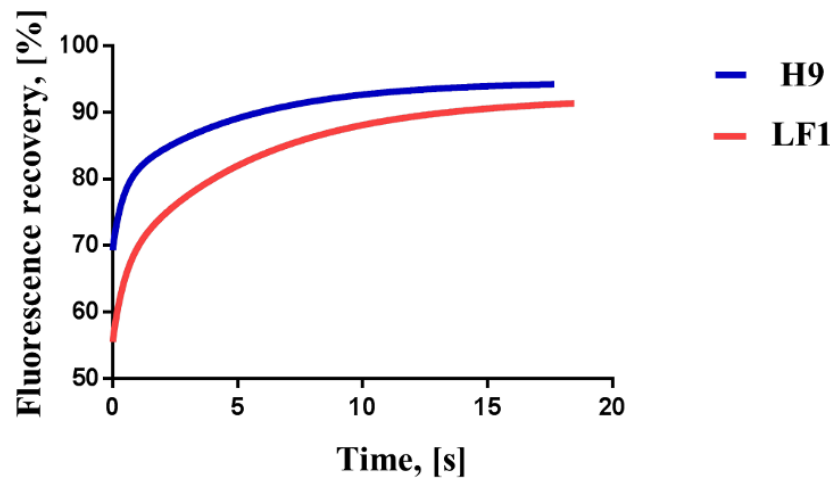

d.

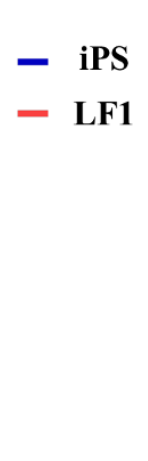

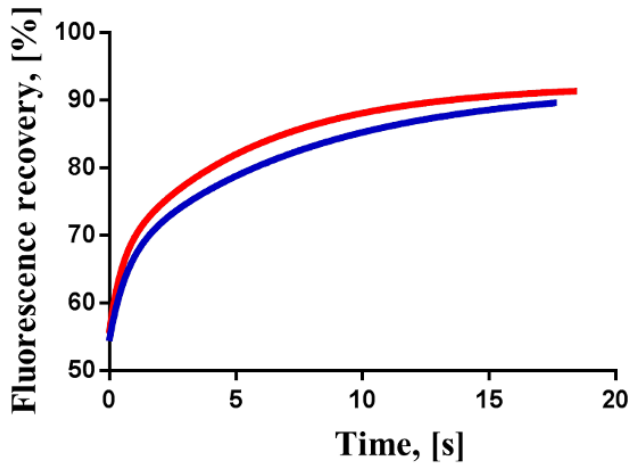

- LF1

- LF1 sen.

Figure $2 \mid$ Pairwise comparison of HP1 $\beta$ FRAP curves from H9 hES, iPS, LF1 and senescent LF1 cells. (a) H9 and iPS recovery curves; the recovery parameters for H9 hES and iPS cells are not significantly different to each other ( $p=0.85$ ). (b) H9 and LF1 recovery curves; the recovery parameters for LF1 cells are significantly different $(p<0.0001)$ from H9 cells $(c)$ iPS and LF1 recovery curves; the recovery parameters for LF1 cells are significantly different $(\mathrm{p}<0.0001)$ from iPS cells. (d) Young LF1 and senescent LF1 recovery curves; the recovery parameters for young LF1 cells are significantly different $(\mathrm{p}<0.0001)$ from senescent LF1 cells. All recovery curves are calculated from at least 3 experiments. For details of the recovery curve parameters please see Table 1; the individual HP1 $\beta$ FRAP measurements are presented in Supplementary Fig. S6. 
a.

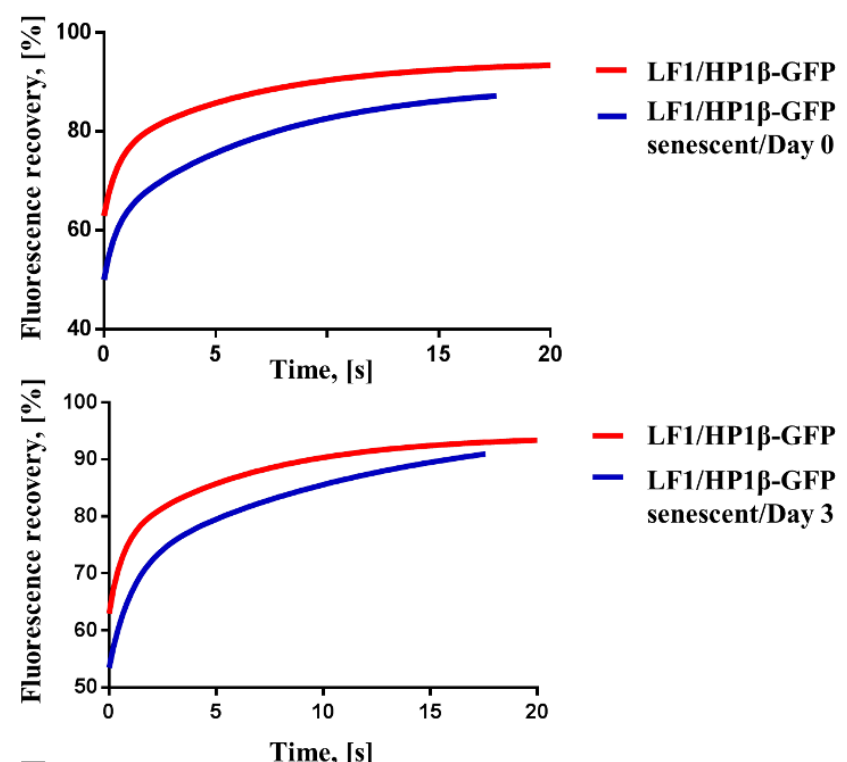

c.

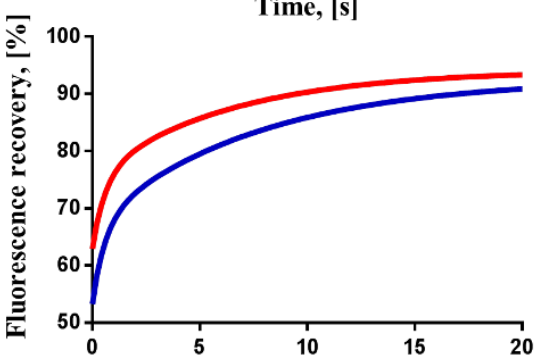

- LF1/HP1ß-GFP

LF1/HP1ß-GFP senescent/Day 6

d.

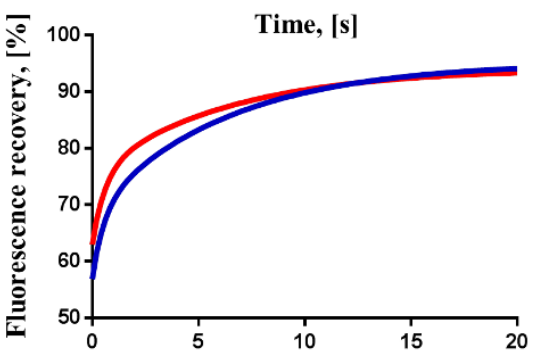

- LF1/HP1ß-GFP

- LF1/HP1ß-GFP senescent/Day 9

e.

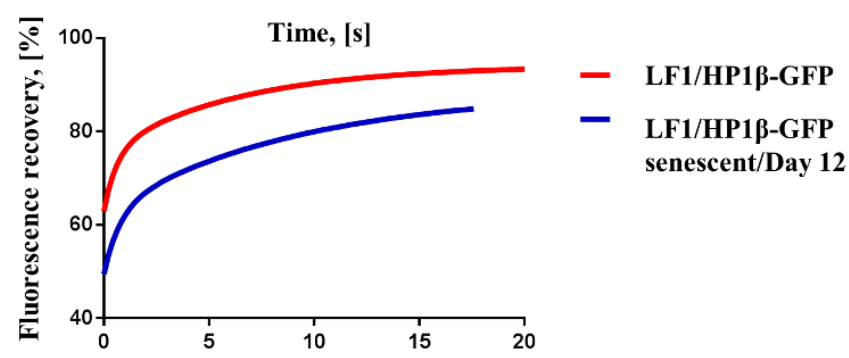

Time, [s]

Figure $3 \mid$ Comparison of HP1 $\beta$ FRAP curves from young LF1/HP1 $\beta-$ GFP cells and senescent LF1/HP $\beta-$ GFP cells undergoing reprogramming. (a) The young and senescent LF1/HP1 $\beta$ - GFP fibroblast recovery curves measured on day 0 of reprogramming; the recovery parameters for young LF1/ HP1 $\beta$ - GFP cells are significantly different $(p<0.0001)$ from senescent LF1/HP1 $\beta-$ GFP cells. (b) The young and senescent LF1/HP1 $\beta-$ GFP fibroblast recovery curves after 3 days of reprogramming; the recovery parameters for young LF1/HP1 $\beta-$ GFP cells are significantly different $(\mathrm{p}<0.0001)$ from senescent LF1/HP1 $\beta$ - GFP cells after 3 days of reprogramming. (c) The young and senescent LF1/HP1 $\beta-$ GFP fibroblast recovery curves after 6 days of reprogramming; the recovery parameters for young LF1/HP1 $\beta$ - GFP cells are significantly different $(\mathrm{p}<0.0001)$ from senescent LF1/HP1 $\beta-$ GFP cells after 6 days of reprogramming. (d) The young and senescent LF1/HP1 $\beta-$ GFP fibroblast recovery curves after 9 days of reprogramming; the recovery parameters for young LF1/HP1 $\beta$ - GFP cells are not significantly different $(\mathrm{p}=0.12)$ from senescent LF1/HP1 $\beta-$ GFP cells after 9 days of reprogramming. (e) The young and senescent LF1/HP1 $\beta-$ GFP fibroblast recovery curves after 12 days of reprogramming; the recovery parameters for young LF1/HP1 $\beta-$ GFP cells are significantly different $(p<0.0001)$ from senescent LF1/HP1 $\beta-$ GFP cells after 12 days of reprogramming. All recovery curves are calculated from at least 3 experiments. For details of the recovery curve parameters please see Table 2 ; the individual HP1 $\beta$ FRAP measurements are presented in Supplementary Fig. S7. 
Table 2 | FRAP analysis of HP1 $\beta$ mobility during reprogramming of senescent LF1/HP1 $\beta$-GFP cells. The left hand column represents the

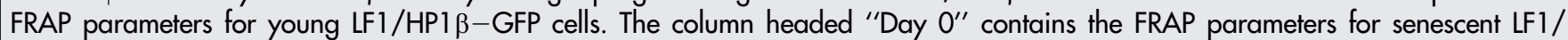
HPI $\beta$-GFP cells just before lipofection with dsRed-OSKML. Days 3, 6, 9 and 12 are the columns containing the parameters for senescent LF1/HP1 $\beta$-GFP cells after 3, 6, 9 and 12 days of reprogramming (i.e., 3, 6, 9 and 12 days after lipofection with dsRed-OSKML). Using these FRAP parameters it can be seen in Figs. $3 a, b$, c and e that the recovery of HP $1 \beta$ in young LF $1 / H P 1 \beta-$ GFP fibroblasts is significantly faster ( $p<0.0001$ ) compared to the recovery of HP1 $\beta$ in Day 0 senescent LF $1 / H P 1 \beta-$ GFP fibroblasts and after 3,6 , and 12 days of reprogramming. There is no significant difference $(p=0.12)$ between the recovery parameters of young LF1/HP1 $\beta-$ GFP fibroblasts and senescent LF1/HP1 $\beta$-GFP fibroblasts on day 9 of reprogramming (recovery curves see Fig. $3 d$ )

\begin{tabular}{lcccccc} 
& LFI $/ \mathrm{HP1} \beta$ & Day 0 & Day 3 & Day 6 & Day 9 \\
\hline $\mathrm{T}_{1 / 2 \text { fast }}(\mathrm{s})$ & $0.4 \pm 0.1$ & $0.37 \pm 0.1$ & $0.7 \pm 0.15$ & $0.4 \pm 0.1$ & $0.38 \pm 0.14$ & $0.5 \pm 0.13$ \\
$\mathrm{~T}_{1 / 2 \text { slow }}(\mathrm{s})$ & $4.4 \pm 0.6$ & $5.1 \pm 0.6$ & $8.3 \pm 2.8$ & $5.2 \pm 0.76$ & $4.4 \pm 0.66$ & $6.4 \pm 1.24$ \\
Free $(\%)$ & $34 \pm 3.4$ & $24 \pm 2.56$ & $37 \pm 2.4$ & $29 \pm 2.7$ & $28 \pm 4.2$ & $27 \pm 2.5$ \\
Bound $(\%)$ & $50 \pm 3.4$ & $55 \pm 2.56$ & $57 \pm 2.4$ & $55 \pm 2.7$ & $61 \pm 4.2$ & $51 \pm 2.5$ \\
$\mathrm{f}_{\text {im }}(\%)$ & $16 \pm 2.3$ & $20 \pm 3.4$ & $6 \pm 8.4$ & $16 \pm 3.9$ & $11 \pm 4.8$ & $22 \pm 4.4$ \\
\hline
\end{tabular}

The increase in HP $1 \beta$ mobility was transient. HP $1 \beta$ mobility slowed and fell back to senescent levels by day 12 of reprogramming. Throughout the 12 days of reprogramming the senescent fibroblasts were maintained in fibroblast medium and they retained their fibroblast morphology; it takes 40 days for iPS cells to be generated from senescent cells and only after transfer to hES medium ${ }^{5}$.

The transient nature of the rejuvenation of HP $1 \beta$ mobility at day 9 is at around the time the epigenome is thought to become "unstable" in specialised cells undergoing reprogramming ${ }^{49,50}$. Instability of the rejuvenated epigenome (including histone/DNA modifications) may be an obstacle to the generation of stably rejuvenated cells. Stabilisation may require the development of culture conditions or chemicals that can maintain the rejuvenated state, by analogy with the regimes that have been developed for the "capture" of the pluripotent state ${ }^{51}$. It is of course possible that the intimate association of developmental reprogramming to age reprogramming does not allow the stabilisation of a rejuvenated state without passage through an embryonic (ES/iPS) stage. It is likely that cell biological processes (organelles), epigenome modifications and molecular pathways in the senescent cells are rejuvenated at different times to others during the reprogramming process and some are more refractory to age reprogramming and require passage through an embryonic (ES/ iPS) stage. The mechanisms and degree of rejuvenation of senescent cells may also be dependent upon how the senescent state was induced; the "senescence" induced by interferon gamma and TNF treatement ${ }^{30}$ may be molecularly different to that induced, for example, by replicative exhaustion. It also remains to be shown that the cell biological processes, epigenome modifications and molecular pathways in a rejuvenated cell are the same as in young cell that has not been through the ageing process. If they are not the same, the ability to "age reprogram" particular aspects of cellular physiology is likely to be facilitated by understanding the novel pathways and mechanisms that operate in rejuvenated cells.

Much work needs to be done to validate age reprogramming as another approach to patient-specific regenerative therapies. But there are clear advantages to pursuing such an alternative to $\mathrm{SCNT}^{52}$ and iPS cell ${ }^{53}$ based therapies. The present work indicates that developmental and age reprogramming may be separated, at least in terms of HP1 $\beta$ mobility, suggesting that such a pursuit could be fruitful.

\section{Methods}

Cells and reagents. LF1 human embryonic fibroblasts were a gift from John Sedivy (Brown University Providence, Rhode Island, USA) and were described previously ${ }^{54}$. Cells were maintained in Ham's F10 medium (PAN-Biotech, Germany) supplemented with 15\% FBS (Life Technologies, Darmstadt, Germany).

H9 hES cells were obtained from Wisconsin International Stem Cell Bank (WiCell Research Institute Inc., Madison, WI). H9 hES and iPS cells were cultured on Matrigel (BD Biosciences, Germany) in mTeSR1 media (STEMCELL Technologies, Grenoble, France).
$\mathrm{HP} 1 \beta$ IgG rat monoclonal $\mathrm{Ab}$ (MAC353), $\mathrm{Me}(3) \mathrm{K} 9 \mathrm{H} 3$ polyclonal $\mathrm{Ab}$ were used as described $^{55,56}$. Ki-67 monoclonal Ab (MIB-1) was from DAKO (Hamburg, Germany). Tra 1-60, Tra1-81, Nanog, Sox2, Pax6 Abs were from Stemgent (Miltenyi Biotec $\mathrm{GmbH}$, Germany). HP1 $\alpha$ (rabbit IgG), HP1 $\gamma$ (rabbit IgG), Histone H3 (mouse IgG), Oct4 (C30A3) Abs and Senescence $\beta$-Galactosidase Staining Kit were from Cell Signaling Technology (New England Biolabs, Germany). Sox 17, Brachyury Abs, BMP4, Activin A were from R\&D (Wiesbaden-Nordenstadt, Germany). c-Myc and Lin 28 polyclonal Abs were from Abcam (Cambridge, UK). Oct4 (C-10) and Klf4 Ab were from Santa Cruz Biotechnology (Heidelberg, Germany). GAPDH Ab (rabbit IgG) was from Sigma (Munich, Germany). Alkaline Phosphatase Staining Kit was from Cell Biolabs Inc. (Biocat, Heidelberg, Germany). SSEA4 Ab, ROCK kinase inhibitor Y27632 and Sodium Butyrate were from MerckMillipore (Schwalbach, Germany). bFGF and secondary fluorescent antibodies labeled with Alexa dyes were purchased from Life Technologies (Darmstadt, Germany).

DNA constructs. pPB-CAG.OSKML and pCMV-hyPBase constructs for iPS reprogramming ${ }^{46}$ were obtained from Wellcome Trust Sanger Institute (Cambridge, UK). dsRed-OSKML was engineered from pPB-CAG.OSKML by the introduction of an IRES - dsRed sequence derived from pMIdsRedExpress2. Specifically, IRES dsRed was excised from pMIdsRedExpress 2 with a double digest with XhoI and SalI enzymes. The resulting IRES - dsRed was sub-cloned into the unique XhoI site in pPB-CAG.OSKML, giving dsRed-OSKML.

EF1/HP1 $\beta$-GFP construct was made by subcloning EF1 promoter into pEGFP$\mathrm{HP} 1 \beta-\mathrm{C} 1$ vector (described $\mathrm{in}^{38}$ ) at $\mathrm{NheI} / \mathrm{NdeI}$ restriction sites after removal of the CMV promoter.

iPS cell reprogramming. LF1 human embryonic fibroblasts at low passage (p3) were seeded at density $0.65 \times 10^{5} /$ well in Matrigel coated 6 well plates. The next day cells were transfected with OSKML and hyPBase vectors ( $2 \mu \mathrm{g}$ each) using Lipofectamine LTX and Plus reagent (Life Technologies, Darmstadt, Germany). On Day 2 cells were put into mTeSR 1 media which was replaced every day. In addition from Day 2 to Day 11 cells mTeSR1 media was supplemented with $0.25 \mathrm{mM}$ Sodium Butyrate. iPS colonies were picked after Day 18 and transferred onto Matrigel coated 24 well plate for expansion.

For FRAP reprogramming studies LF1/HP1 $\beta$-GFP fibroblasts were plated on $35 \mathrm{~mm}$ ibidi microscopy dishes (ibidi GmbH, Martinsried, Germany) at density $1 \times$ $10^{5} /$ dish. The next day cells were transfected with with dsRed-OSKML and hyPBase vectors $(2 \mu \mathrm{g}$ each) using Lipofectamine LTX and Plus reagent. Transfection efficiency was $44 \pm 11 \%$ (Figure S5b). Cells were maintained in LF1 media (Ham's F10 with $15 \%$ FBS) throughout the whole experiment. Before imaging cell media was replaced for Phenol Red free Ham's F10, 1\% FBS and 20 mM HEPES.

Alkaline phosphatase staining. The assay was performed using Alkaline Phosphatase Staining Kit from Cell Biolabs Inc. (Biocat, Heidelberg, Germany) according to the manufacturer's recommendations.

SA $\boldsymbol{\beta}$-galactosidase staining. The assay was performed using Senescence-associated $\beta$-Galactosidase Staining Kit from Cell Signaling Technology (New England Biolabs, Germany) according to the manufacturer's recommendations. On day 0 and day 9 we determined the $\%$ SA $\beta$-Galactosidase positive \pm s.d. on a slide by counting the number SA $\beta$-Galactosidase positive in 5 fields each of the dimension of $2062.5 \mu \mathrm{m} \times$ $1562.5 \mu \mathrm{m}$ (area $3.22 \mathrm{~mm}^{2}$ ). 100 cells were counted in each field.

Karyotyping. Metaphase chromosome spreads and karyotyping analysis was performed at the Intitute of Human Genetics University Clinic Freiburg.

Bisulfite sequencing analysis. Isolation of DNA from H9 hES, iPS and LF1 cells was performed using DNeasy kit (Qiagen, Germany). Samples were analysed for DNA methylation by pyrosequencing analysis at EpigenDx Inc. (Hopkinton, MA, USA). Methylation levels were analysed at $7 \mathrm{CpGs}$ for $\operatorname{Oct} 4(-2234,-2140,+5,+31,+41$, 
+59 from Oct4 transcriptional start site (TSS)) and at 2 CpGs for $\operatorname{Nanog}(-346$ and -337 from Nanog TSS) genes.

Differentiation into germ layers. For ectoderm differentiation iPS cells were dissociated using Accutase and seeded onto Matrigel coated ibidi dishes (ibidi GmbH, Martinsried, Germany) in STEMdiff Neural Induction Medium (STEMCELL Technologies, Grenoble, France) supplemented with $10 \mu \mathrm{M}$ ROCK inhibitor. The next day medium was replaced with STEMdiff Neural Induction Medium without ROCK inhibitor and subsequently was changed daily for 10 days. On Day 11 cells were stained by immunofluorescence for Pax6 ectoderm marker.

Differentiation into mesoderm layer was performed as following. Cells were passaged as clumps using Dispase onto Matrigel coated ibidi dish (ibidi GmbH, Martinsried, Germany) in mTeSR1 media. The next day cells were fed with Apel Medium (STEMCELL Technologies, Grenoble, France) supplemented with $0.5 \mathrm{ng} /$ ml BMP4. On Day 2 Apel Medium was supplemented with $10 \mathrm{ng} / \mathrm{ml} \mathrm{BMP4,} 10 \mathrm{ng} /$ $\mathrm{ml}$ Activin A and $10 \mathrm{ng} / \mathrm{ml}$ bFGF. On Day 3 medium was replaced with Apel Medium containing $10 \mathrm{ng} / \mathrm{BMP} 4,10 \mathrm{ng} / \mathrm{ml}$ Activin A and $5 \mathrm{ng} / \mathrm{ml}$ bFGF. Immunofluorescence staining for mesoderm marker Brachyury was performed on day 4 .

iPS cells were differentiated into endoderm germ layer using STEMdiff Definitive Endoderm kit (STEMCELL Technologies, Grenoble, France) according to the manufacturer's instructions. On day 5 of differentiation cells were fixed and stained for the Sox17 endoderm marker.

Transfection. Transient transfection of LF1 cells was done using Lipofectamine LTX and Plus reagent (Life Technologies, Darmstadt, Germany). Cells were plated on $35 \mathrm{~mm}$ ibidi dishes (ibidi GmbH, Martinsried, Germany) at a density of $1.5 \times 10^{5}$ / dish. $24 \mathrm{~h}$ later, cells were transiently transfected with $1 \mu \mathrm{g}$ of HP1 $\beta$-GFP plasmid according to the manufacturer's recommendations. $48 \mathrm{~h}$ later cells were imaged at the confocal microscope in Phenol Red free Ham's F10, 1\% FBS and 20 mM HEPES.

Transfections of H9 hES and iPS cells were done using Stemfect Enhanced hES transfection kit (Miltenyi Biotec $\mathrm{GmbH}$, Germany). Cells were passaged as clumps using dispase onto Matrigel coated ibidi dishes (ibidi GmbH, Martinsried, Germany) in mTeSR1 media. $24 \mathrm{~h}$ later, cells were transiently transfected with $13 \mu \mathrm{g}$ of HP1 $\beta$ GFP plasmid according to the manufacturer's recommendations. $48 \mathrm{~h}$ later cells were imaged at the confocal microscope in Phenol Red free DMEM (Life Technologies).

LF1 cells expressing HP1及. Transfection of LF1 cells was done using Amaxa Nucleofection (Lonza, Germany). $1 \times 10^{6}$ cells were transfected with $1 \mu \mathrm{g}$ of HP1 $\beta$ GFP plasmid per $100 \mu$ Ingenio electroporation solution (Mirius Bio LLC, WI, USA) in $0.2 \mathrm{~cm}$ cuvettes with U023 program settings according to the manufacturer's recommendations. After $72 \mathrm{~h}$ cells were cultured in the presence of G418 [400 $\mu \mathrm{g} / \mathrm{ml}]$ (Life Technologies) until control un-transfected cells were completely eliminated. Subsequently HP1 $\beta$-GFP cells were flow sorted for different fractions with low, medium and high levels of HP1 $\beta$-GFP expression. Cells were maintained in culture in the presence of $\mathrm{G} 418[80 \mu \mathrm{g} / \mathrm{ml}]$.

Immunoblotting. Cell lysates were prepared in RIPA lysis buffer supplemented with protease inhibitors. Protein concentrations were determined by BCA assay (Pierce, Thermo Scientific). Cell lysates were separated by SDS-PAGE and transferred onto nitrocellulose membrane (Millipore). Proteins were stained with primary and secondary Abs and detected by ECL (Pierce, Thermo Scientific). Chemiluminescence was detected by Fusion Fx7 Imaging System (Peqlab, Germany).

Immunofluorescence staining. Cells were seeded on ibidi $35 \mathrm{~mm}$ tissue culture dishes (ibidi GmbH, Martinsried, Germany) at $1.5 \times 10^{5} /$ well. Cells were fixed with $4 \%$ paraformaldehyde for $20 \mathrm{~min}$ at RT and permeabilized with $0.05 \%$ Triton X-100. After $1 \mathrm{~h}$ blocking staining with specific Abs was performed for $1 \mathrm{~h} \mathrm{RT}$ or overnight at $4{ }^{\circ} \mathrm{C}$. After washing cells were incubated with fluorescent secondary Abs for $1 \mathrm{~h}$ RT. Nuclei were stained with Hoechst during the washing steps. Cells were mounted in Pro Long Gold mounting media (Life Technologies).

FRAP analysis and confocal microscopy. Confocal imaging was performed with Zeiss LSM 5 Live DUO inverted confocal microscope using LCI- Plan Neofluar $63 \times /$ 1.3 DIC immersion corr. objective or LD LCI Plan Apochromat $25 \times / 0.8$ immersion corr. objective. FRAP experiments were performed using LCI- Plan Neofluar $63 \times / 1.3$ DIC immersion corr. objective and 488 laser line with a LF505 emission filter for GFP. A $1 \mu \mathrm{m}^{2}$ circular ROI within nucleus in heterochromatic region was set up for bleaching, $4 \mu \mathrm{m}^{2}$ ROI for bleaching correction, and additionally $4 \mu \mathrm{m}^{2}$ control ROI outside of cell area was acquired for background subtraction. The laser intensity was set up at $75 \%$. Ten images were taken before the bleach pulse (15 laser iterations with bleaching intensity output $100 \%$ ) and 60 images were taken after the bleaching with image acquisition each $0.3 \mathrm{~s}$ with $5 \%$ laser transmission. All FRAP experiments were performed in temperature controlled $\mathrm{CO}_{2}$ supplied chamber. All data were background subtracted and normalized using Excel software and analyzed by nonlinear regression analysis with GraphPad Prism Software (San Diego, USA). Recovery parameters were estimated from the fitted recovery curves.

Statistics. When indicated experimental data (minimum of three biological replicates) was analyzed for statistical significance employing the Extra sum-ofsquares F-test with GraphPad Prism Software (San Diego, USA). Significance is described in figure legends. All western blots and confocal images are representatives of biological replicates.
1. Gurdon, J. B. The cloning of a frog. Development 140, 2446-8 (2013)

2. Wilmut, I., Schnieke, A. E., McWhir, J., Kind, A. J. \& Campbell, K. H. Viable offspring derived from fetal and adult mammalian cells. Nature 385, 810-3 (1997).

3. Mizutani, E., Ono, T., Li, C., Maki-Suetsugu, R. \& Wakayama, T. Propagation of senescent mice using nuclear transfer embryonic stem cell lines. Genesis $\mathbf{4 6}$, 478-83 (2008).

4. Takahashi, K. \& Yamanaka, S. Induction of pluripotent stem cells from mouse embryonic and adult fibroblast cultures by defined factors. Cell 126, 663-76 (2006).

5. Lapasset, L. et al. Rejuvenating senescent and centenarian human cells by reprogramming through the pluripotent state. Genes \& Development 25, 2248-2253 (2011).

6. Rivera, C. M. \& Ren, B. Mapping human epigenomes. Cell 155, 39-55 (2013).

7. Bickmore, W. A. \& van Steensel, B. Genome architecture: domain organization of interphase chromosomes. Cell 152, 1270-84 (2013).

8. Singh, P. B. \& Zacouto, F. Nuclear reprogramming and epigenetic rejuvenation. J Biosci 35, 315-9 (2010).

9. Manukyan, M. \& Singh, P. B. Epigenetic rejuvenation. Genes Cells 17, 337-43 (2012).

10. Shah, P. P. et al. Lamin B1 depletion in senescent cells triggers large-scale changes in gene expression and the chromatin landscape. Genes Dev 27, 1787-99 (2013).

11. Lopez-Otin, C., Blasco, M. A., Partridge, L., Serrano, M. \& Kroemer, G. The hallmarks of aging. Cell 153, 1194-217 (2013).

12. Brown, S. W. Heterochromatin. Science 151, 417-25 (1966).

13. Bulut-Karslioglu, A. et al. A transcription factor-based mechanism for mouse heterochromatin formation. Nat Struct Mol Biol 19, 1023-30 (2012).

14. Kourmouli, N. et al. Heterochromatin and tri-methylated lysine 20 of histone H4 in animals. J Cell Sci 117, 2491-501 (2004).

15. Schotta, G. et al. A silencing pathway to induce $\mathrm{H} 3-\mathrm{K} 9$ and $\mathrm{H} 4-\mathrm{K} 20$ trimethylation at constitutive heterochromatin. Genes Dev 18, 1251-62 (2004).

16. Chen, T., Tsujimoto, N. \& Li, E. The PWWP domain of Dnmt3a and Dnmt3b is required for directing DNA methylation to the major satellite repeats at pericentric heterochromatin. Mol Cell Biol 24, 9048-58 (2004).

17. Wallrath, L. L. \& Elgin, S. C. Position effect variegation in Drosophila is associated with an altered chromatin structure. Genes Dev 9, 1263-77 (1995).

18. Casanova, M. et al. Heterochromatin Reorganization during Early Mouse Development Requires a Single-Stranded Noncoding Transcript. Cell Rep 4, 1156-67 (2013)

19. Grewal, S. I. \& Elgin, S. C. Transcription and RNA interference in the formation of heterochromatin. Nature 447, 399-406 (2007)

20. Billur, M., Bartunik, H. D. \& Singh, P. B. The essential function of HP1 beta: a case of the tail wagging the dog? Trends Biochem Sci 35, 115-23 (2010).

21. Grewal, S. I. \& Elgin, S. C. Heterochromatin: new possibilities for the inheritance of structure. Curr Opin Genet Dev 12, 178-87 (2002).

22. Maison, C. \& Almouzni, G. HP1 and the dynamics of heterochromatin maintenance. Nat Rev Mol Cell Biol 5, 296-304 (2004).

23. Jones, D. O., Cowell, I. G. \& Singh, P. B. Mammalian chromodomain proteins: their role in genome organisation and expression. Bioessays 22, 124-37 (2000).

24. Singh, P. B. HP1 proteins--what is the essential interaction? Genetika 46, 1424-9 (2010).

25. de Lange, T. How shelterin solves the telomere end-protection problem. Cold Spring Harb Symp Quant Biol 75, 167-77 (2010).

26. Marion, R. M. \& Blasco, M. A. Telomere rejuvenation during nuclear reprogramming. Curr Opin Genet Dev 20, 190-6 (2010).

27. Narita, M. et al. Rb-mediated heterochromatin formation and silencing of E2F target genes during cellular senescence. Cell 113, 703-16 (2003).

28. Sadaie, M. et al. Redistribution of the Lamin B1 genomic binding profile affects rearrangement of heterochromatic domains and SAHF formation during senescence. Genes Dev 27, 1800-8 (2013).

29. Gorgoulis, V. G. \& Halazonetis, T. D. Oncogene-induced senescence: the bright and dark side of the response. Curr Opin Cell Biol 22, 816-27 (2010).

30. Braumuller, H. et al. T-helper-1-cell cytokines drive cancer into senescence. Nature 494, 361-5 (2013).

31. Sikora, E., Arendt, T., Bennett, M. \& Narita, M. Impact of cellular senescence signature on ageing research. Ageing Res Rev 10, 146-52 (2011).

32. Kwon, S. H. \& Workman, J. L. The changing faces of HP1: From heterochromatin formation and gene silencing to euchromatic gene expression: HP1 acts as a positive regulator of transcription. Bioessays 33, 280-9 (2011).

33. Feldman, N. et al. G9a-mediated irreversible epigenetic inactivation of Oct-3/4 during early embryogenesis. Nat Cell Biol 8, 188-94 (2006).

34. Sridharan, R. et al. Proteomic and genomic approaches reveal critical functions of $\mathrm{H} 3 \mathrm{~K} 9$ methylation and heterochromatin protein-1gamma in reprogramming to pluripotency. Nat Cell Biol 15, 872-82 (2013).

35. Chambers, I. et al. Functional expression cloning of Nanog, a pluripotency sustaining factor in embryonic stem cells. Cell 113, 643-55 (2003).

36. Mitsui, K. et al. The homeoprotein Nanog is required for maintenance of pluripotency in mouse epiblast and ES cells. Cell 113, 631-42 (2003).

37. Dundr, M. \& Misteli, T. Measuring dynamics of nuclear proteins by photobleaching. Curr Protoc Cell Biol 18, 13.5.1-13.5.18 (2003).

38. Cheutin, T. et al. Maintenance of stable heterochromatin domains by dynamic HP1 binding. Science 299, 721-5 (2003). 
39. Festenstein, R. et al. Modulation of heterochromatin protein 1 dynamics in primary Mammalian cells. Science 299, 719-21 (2003).

40. Cheutin, T., Gorski, S. A., May, K. M., Singh, P. B. \& Misteli, T. In vivo dynamics of Swi6 in yeast: evidence for a stochastic model of heterochromatin. Mol Cell Biol 24, 3157-67 (2004).

41. Schmiedeberg, L., Weisshart, K., Diekmann, S., Meyer Zu Hoerste, G. \& Hemmerich, P. High- and low-mobility populations of HP1 in heterochromatin of mammalian cells. Mol Biol Cell 15, 2819-33 (2004).

42. Dialynas, G. K. et al. Methylation-independent binding to histone $\mathrm{H} 3$ and cell cycle-dependent incorporation of HP1beta into heterochromatin. J Biol Chem 281, 14350-60 (2006).

43. Dialynas, G. K. et al. Plasticity of HP1 proteins in mammalian cells. J Cell Sci 120, 3415-24 (2007).

44. Muller, K. P. et al. Multiscale analysis of dynamics and interactions of heterochromatin protein 1 by fluorescence fluctuation microscopy. Biophys $J \mathbf{9 7}$, 2876-85 (2009).

45. Meshorer, E. et al. Hyperdynamic plasticity of chromatin proteins in pluripotent embryonic stem cells. Dev Cell 10, 105-16 (2006).

46. Yusa, K., Rad, R., Takeda, J. \& Bradley, A. Generation of transgene-free induced pluripotent mouse stem cells by the piggyBac transposon. Nat Methods 6, 363-9 (2009).

47. Bowman, P. D., Meek, R. L. \& Daniel, C. W. Aging of human fibroblasts in vitro. Correlations between DNA synthetic ability and cell size. Exp Cell Res 93, 184-90 (1975).

48. Suhr, S. T. et al. Mitochondrial rejuvenation after induced pluripotency. PLoS One 5, e14095 (2010)

49. Nagy, A. \& Nagy, K. The mysteries of induced pluripotency: where will they lead? Nat Methods 7, 22-4 (2010).

50. Efe, J. A. et al. Conversion of mouse fibroblasts into cardiomyocytes using a direct reprogramming strategy. Nat Cell Biol 13, 215-222 (2011).

51. Buehr, M. et al. Capture of authentic embryonic stem cells from rat blastocysts. Cell 135, 1287-98 (2008).

52. Tachibana, M. et al. Human embryonic stem cells derived by somatic cell nuclear transfer. Cell 153, 1228-38 (2013).

53. Yamanaka, S. Strategies and new developments in the generation of patientspecific pluripotent stem cells. Cell Stem Cell 1, 39-49 (2007).
54. Brown, J. P., Wei, W. \& Sedivy, J. M. Bypass of senescence after disruption of p21CIP1/WAF1 gene in normal diploid human fibroblasts. Science 277, 831-4 (1997).

55. Wreggett, K. A. et al. A mammalian homologue of Drosophila heterochromatin protein 1 (HP1) is a component of constitutive heterochromatin. Cytogenet Cell Genet 66, 99-103 (1994).

56. Cowell, I. G. et al. Heterochromatin, HP1 and methylation at lysine 9 of histone H3 in animals. Chromosoma 111, 22-36 (2002).

\section{Acknowledgments}

This study was supported by the Excellence Initiative of the German Federal and State Governments (EXC 294). We would like to thank Dr.M. Leipoldt for karyotyping analysis.

\section{Author contributions}

M.M., P.B.S. conceived the experiments. M.M. designed and performed the experiments, and analysed the data. M.M., P.B.S. discussed the data and wrote the paper.

\section{Additional information}

Supplementary information accompanies this paper at http://www.nature.com/ scientificreports

Competing financial interests: The authors declare no competing financial interests.

How to cite this article: Manukyan, M. \& Singh, P.B. Epigenome rejuvenation: HP1 $\beta$ mobility as a measure of pluripotent and senescent chromatin ground states. Sci. Rep. 4, 4789; DOI:10.1038/srep04789 (2014).

This work is licensed under a Creative Commons Attribution-NonCommercialShareAlike 3.0 Unported License. The images in this article are included in the article's Creative Commons license, unless indicated otherwise in the image credit; if the image is not included under the Creative Commons license, users will need to obtain permission from the license holder in order to reproduce the image. To view a copy of this license, visit http://creativecommons.org/licenses/by-nc-sa/3.0/ 